\title{
Anisotropic deformation of extruded magnesium alloy AZ31 under uniaxial compression: A study with in situ synchrotron x-ray imaging and diffraction
}

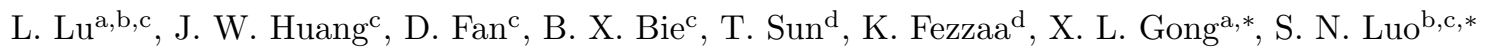 \\ ${ }^{a}$ CAS Key Laboratory of Mechanical Behavior and Design of Materials, Department of Modern Mechanics, University of Science and \\ Technology of China, Hefei, Anhui 230027, P. R. China \\ ${ }^{b}$ Key Laboratory of Advanced Technologies of Materials, Ministry of Education, Southwest Jiaotong University, Chengdu, Sichuan 610031, \\ P. R. China \\ ${ }^{c}$ The Peac Institute of Multiscale Sciences, Chengdu, Sichuan 610031, P. R. China \\ ${ }^{d}$ Advanced Photon Source, Argonne National Laboratory, Argonne, IL 60439, USA
}

\begin{abstract}
In situ synchrotron x-ray imaging and diffraction are used to investigate anisotropic deformation of an extruded magnesium alloy AZ31 under uniaxial compression along two different directions, with the loading axis (LA) either parallel or perpendicular to the extrusion direction (ED), referred to as LA $\|$ ED and $L A \perp E D$, respectively. Multiscale measurements including stress-strain curves (macroscale), x-ray digital image correlation (mesoscale), and diffraction (microscale) are obtained simultaneously. Electron backscatter diffraction is performed on samples collected at various strains to characterize deformation twins. The rapid increase in strain hardening rate for the LA $\|$ ED loading is attributed to marked $\{10 \overline{1} 2\}$ extension twinning and subsequent homogenization of deformation, while dislocation motion leads to inhomogeneous deformation and a decrease in strain hardening rate.
\end{abstract}

Keywords: Magnesium alloys, Twinning, Dislocation, Digital image correlation, XRD

\section{Introduction}

Magnesium alloys show a remarkable potential as structural components for their low density, high specific stiffness and high specific strength $[1,2]$. However, wide applications of magnesium alloys are hindered by their poor formability at room temperature, and overcoming such a deficiency requires a better understanding of deformation mechanisms, and microstructural effects on mechanical properties of these alloys.

Magnesium has a hexagonal close-packed structure, with possible deformation modes including (0001) $\langle a\rangle$ basal slip, $\{10 \overline{1} 0\}\langle a\rangle$ prismatic slip, $\{10 \overline{1} 1\}\langle a\rangle$ and $\{11 \overline{2} 2\}\langle c+a\rangle$ pyramidal slips [3-5], as well as $\{10 \overline{1} 2\}$ extension and $\{10 \overline{1} 1\}$ contraction twinning [6-11]. Given the special deformation modes associated with dislocation motion and deformation twinning, a strong anisotropy in deformation is characteristic of magnesium and its alloys [12, 13]. Similar to magnesium, titanium with a hexagonal close-packed structure also shows anisotropy in deformation [14, 15]. The stress-strain curves for different loading directions exhibit drastic differences. For compression perpendicular to the $c$-axis, three distinct regimes (I-III) are observed

\footnotetext{
* Corresponding author

Email addresses: gongxl@ustc.edu.cn (X. L. Gong), sluo@pims.ac.cn; sluo@swjtu.cn (S. N. Luo)
}

[16-21]. In regime II, strain hardening rate shows a pronounced rise, while a decrease in strain hardening rates occurs in regimes I and III. The variations of strain hardening rates were explained with deformation twinning and dislocation motion [17-29], but the exact nature is still under debate.

Jiang et al. [26] suggested that intersections between primary and secondary $\{10 \overline{1} 2\}$ extension twins in regime II lead to significant grain refinement and subsequently an increase in strain hardening rate. By contrast, without resorting to the Hall-Petch effect [30, 31], Oppedal et al. [27] used a viscoplastic self-consistent model [32, 33] to show that rotation to a hard orientation via twinning is sufficient to induce increasing hardening rate. Moreover, Barnett et al. [24] proposed that the formation of low angle boundaries arising from the glissile-to-sessile transition of dislocations can lead to such an increase. Wang et al. [20] attributed the increase to texture hardening which leads to grain rotation toward hard orientations by $\{10 \overline{1} 2\}$ extension twinning. Knezevic et al. [17] argued that extension twins in AZ31 are not effective in increasing strain hardening rate, while contraction twins are effective by restricting the slip length associated with pyramidal $\langle c+a\rangle$ slip. However, most conclusions were based on the microstructure of samples deformed at various strains, and real-time, in situ, measurements of mesoscopic strain fields and structure evolution are rare. 
(a)

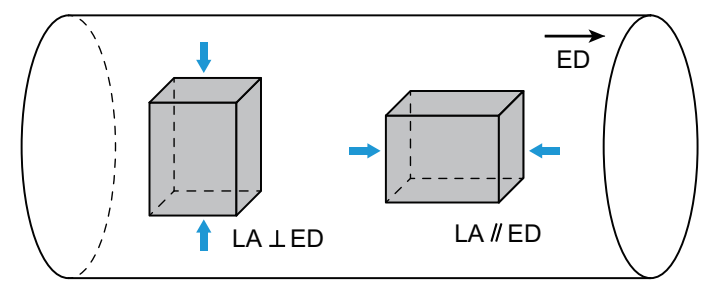

(b)

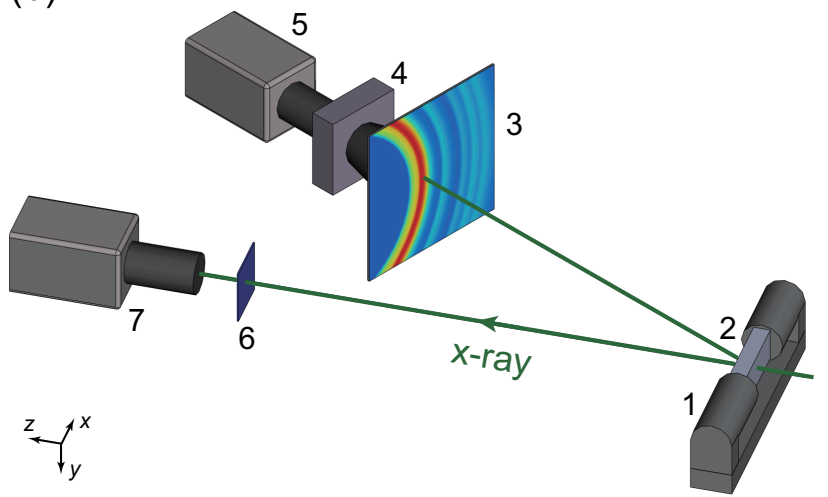

Figure 1: (a) Illustration of the relative orientation of the loading axis (LA) with respect to the extrusion direction (ED). (b) Schematic of the experimental setup for simultaneous x-ray diffraction and imaging under uniaxial compression, along with the coordinate system $(x y z)$. 1: loading device (miniature MTS); 2: sample; 3: diffraction scintillator (with illustrative diffraction rings of magnesium); 4: microchannel plate; 5: diffraction camera; 6: imaging scintillator; 7 : imaging camera.

In this work, uniaxial compression experiments are conducted to investigate the effect of loading direction on strain hardening rate of an extruded magnesium alloy AZ31, using in situ synchrotron x-ray diffraction and imaging. Simultaneous multiscale measurements on macroscopic mechanical properties, mesoscopic strain fields, and microstructure evolution are achieved in terms of stressstrain curves, or with $\mathrm{x}$-ray digital image correlation (XDIC) [34] and x-ray diffraction. Stress-strain curves, and the evolutions of strain fields and diffraction patterns show pronounced anisotropy during uniaxial compression. Electron back-scatter diffraction (EBSD) is conducted to characterize deformation twins. The $\{10 \overline{1} 2\}$ extension twinning tends to homogenize strain fields, and thus effectively boosts strain hardening rate, while dislocation motion leads to inhomogeneous deformation and a decrease in strain hardening rate.

\section{Materials and experiments}

A commercial extruded AZ31 magnesium alloy, consisting of $3.1 \mathrm{wt} \% \mathrm{Al}, 0.9 \mathrm{wt} \% \mathrm{Zn}, 0.4 \mathrm{wt} \% \mathrm{Mn}$, and 95.6 wt $\% \mathrm{Mg}$, is used for uniaxial compression tests. Cuboidal specimens as illustrated in Fig. 1(a) are harvested from an as-received AZ31 bar using electrical discharge machining, and used for simultaneous x-ray imaging and diffraction
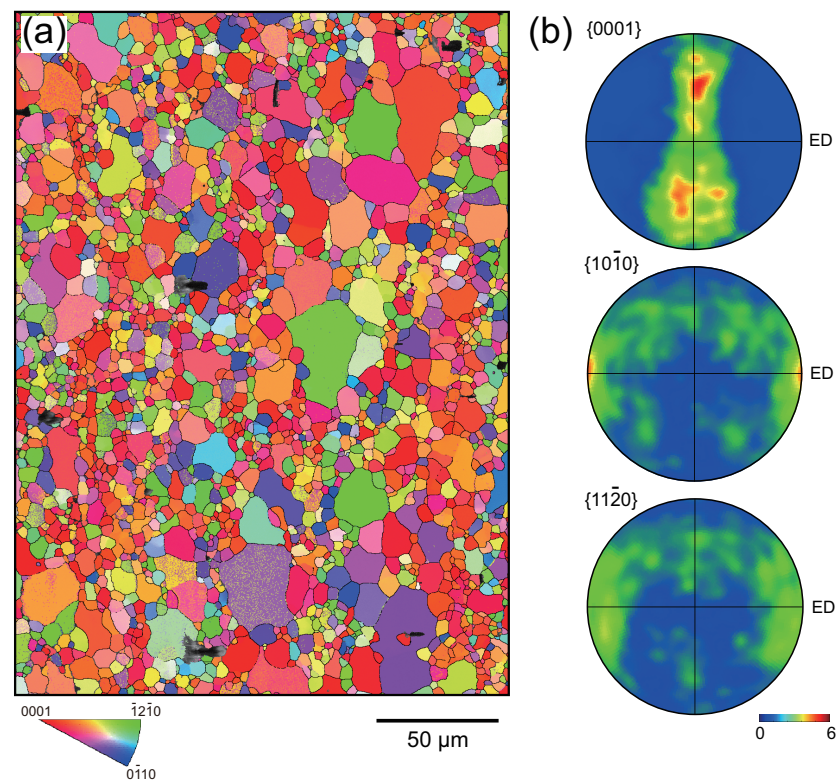

Figure 2: (a) Inverse pole figure map, and (b) pole figures of asextruded magnesium alloy AZ31, viewed along the extrusion direction. The $c$-axes of red grains are perpendicular to ED.

measurements during compression. In order to produce $\mathrm{x}-$ ray speckles for XDIC analysis, the specimens are etched with a solution of $20 \%$ perchloric acid (by volume) and $80 \%$ ethanol for 30 seconds. The dimensions of the sample perpendicular to the $\mathrm{x}$-ray beam direction are $2.6 \times 2.0$ $\mathrm{mm}^{2}$, and the thickness along the $\mathrm{x}$-ray beam direction is $1.0 \mathrm{~mm}$. Two kinds of compression tests are conducted: the loading axis (LA) is either parallel or perpendicular to the extrusion direction (ED), referred to as LA $\| \mathrm{ED}$ and $\mathrm{LA} \perp \mathrm{ED}$, respectively (Fig. 1(a)).

Quasi-static compression tests are performed at room temperature using a home-made miniature material test system (MTS) at beamline 32-ID-B of the Advanced Photon Source. The wavelength of the probe synchrotron x-rays is centered at $0.5 \AA$. The experimental setup is shown in Fig. 1(b) for simultaneous, multiframe, transien$\mathrm{t} x$-ray diffraction and phase contrast imaging measurements under uniaxial compression. The miniature MTS (1 in Fig. 1(b)) loads a specimen (2) at a strain rate of $5 \times 10^{-4} \mathrm{~s}^{-1}$. The $\mathrm{x}$-rays transmitted through the sample are incident on an imaging scintillator $\mathrm{Lu}_{3} \mathrm{Al}_{5} \mathrm{O}_{12}$ or LuAG:Ce (6), and the scattered x-rays, on a diffraction scintillator $\mathrm{Lu}_{2-2 x} \mathrm{Y}_{2 x} \mathrm{SiO}_{5}$ or LYSO:Ce (3). Demagnifying lenses are placed after the diffraction scintillator to increase the effective $q$-range ( $q$ : momentum transfer) sampled by the detector. A microchannel plate (4) is used to intensify the optical signals converted from the scattered $\mathrm{x}$-rays, and synchronized with the diffraction camera (5). The recording cameras for both imaging (7) and diffraction (5) are Photron Fastcam SA-Z. The exposure times for both measurements are $1 \mu \mathrm{s}$, and the frame intervals are $10 \mathrm{~s}$. The HiSPoD program [35] is utilized to index the diffraction rings and convert them into one-dimensional 
(a)

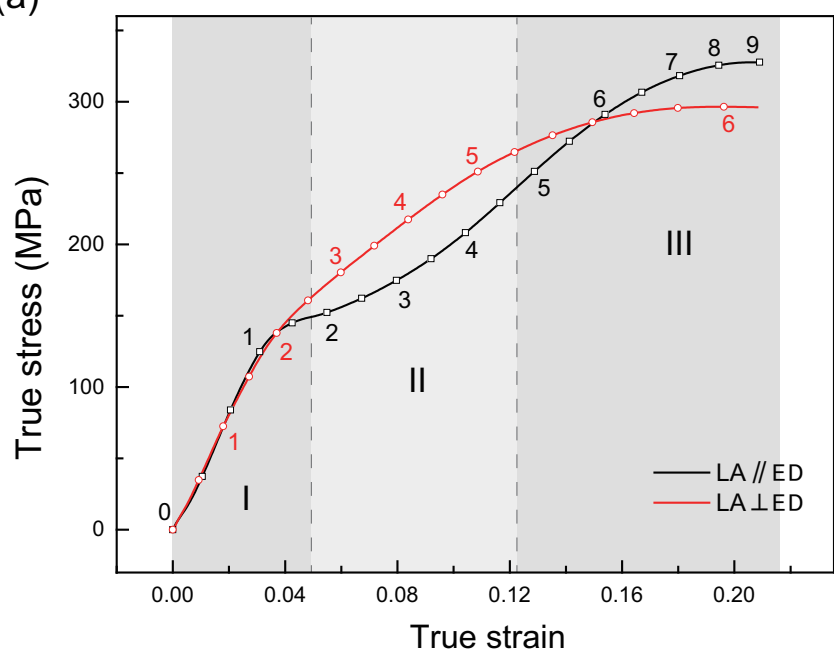

(b)

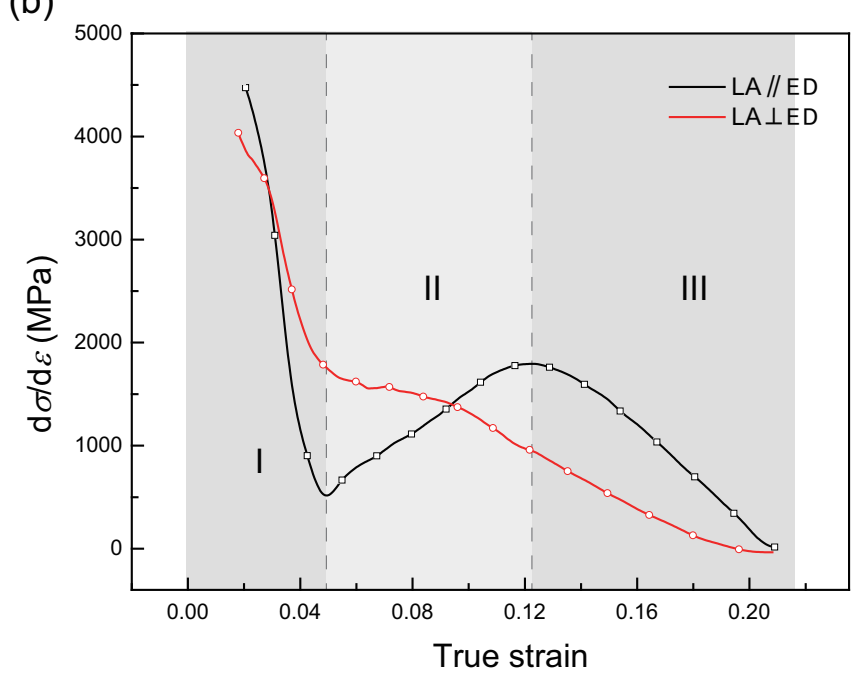

Figure 3: (a) True stress-strain curves, and (b) strain hardening rate versus true strain curves for two different loading directions, LA $\| \mathrm{ED}$ and $\mathrm{LA} \perp \mathrm{ED}$. The symbols (20 seconds apart) denote the instants for simultaneous imaging and diffraction measurements. Numbers 0-9 represent $t_{0}-t_{9}$. The curves are divided into three regimes, I-III.

diffraction pattern (intensity versus $2 \theta$ ) by azimuthal integral. Similar experimental details were presented elsewhere [36]. Samples subjected to different true strains $(\varepsilon=0.055,0.080,0.117)$ are collected for ex situ EBSD analysis of deformation features such as texture and twins.

For EBSD analysis, the as-received and postmortem samples are sectioned parallel to the loading direction, mechanically ground and polished with $2 \mu \mathrm{m}$ and $0.5 \mu \mathrm{m}$ diamond particles, and then electro-polished in $10 \%$ perchloric acid and $90 \%$ ethanol at 5 volts, with a copper rod and the sample as electrodes. EBSD characterization, as well as scanning electron microscope (SEM) analysis, is performed in a FEI Quanta 250 FEG-SEM equipped with Oxford EBSD detector and HKL Channel 5 software. EB$\mathrm{SD}$ scan is performed on a section normal to ED with (a)

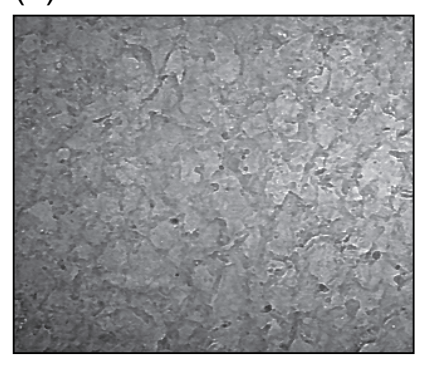

(b)

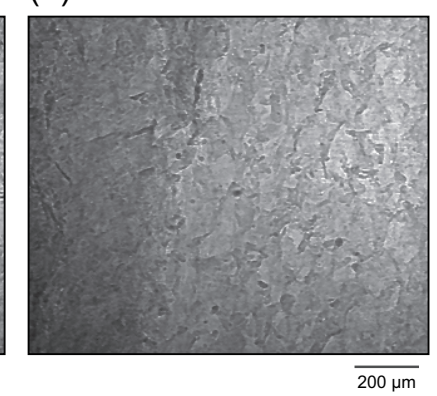

Figure 4: X-ray phase contrast images acquired before (a) and during (b) loading. Shear fracture occurs in (b).

a $25 \mathrm{kV}$ voltage, $15 \mathrm{~mm}$ working distance, and $70^{\circ}$ tilt. Fig. 2 shows the initial microstructure and texture of the as-received AZ31 magnesium alloy obtained from EBSD analysis. Its grain size ranges from $3 \mu \mathrm{m}$ to $50 \mu \mathrm{m}$, and a small amount of second phase $\left(\beta-\mathrm{Mg}_{17} \mathrm{Al}_{12}\right)$ particles are "randomly" distributed within the matrix. The crystallographic $c$-axes in most grains are perpendicular to the extrusion direction.

\section{Experimental results}

\subsection{Macroscale mechanical behavior}

Representative true stress-strain $(\sigma-\varepsilon)$ curves and corresponding strain hardening rates for the two loading directions, $\mathrm{LA} \| \mathrm{ED}$ and $\mathrm{LA} \perp \mathrm{ED}$, demonstrate strong anisotropy in deformation (Fig. 3). The $\sigma-\varepsilon$ curve for the LA $\|$ ED loading exhibits a sigmoidal shape, while that for the $\mathrm{LA} \perp \mathrm{ED}$ loading displays a convex shape (Fig. 3(a)). Such features have long been observed [1721, 24-27, 37, 38]. The yield stresses along both compression directions are similar $(\sim 125 \mathrm{MPa})$.

The strain hardening rate versus true strain curves, $\mathrm{d} \sigma / \mathrm{d} \varepsilon-\varepsilon$, derived from the stress-strain curves, demonstrate three regimes for both loading directions, which also show pronounced differences (Fig. 3(b)). In the elastic stage, strain hardening rate values are the highest for both loading directions. Upon initial yield, the rate decreases rapidly as expected for an elastic-plastic transition (regime I). Then, the strain hardening rate for the LA loading experiences a distinct increase followed by a sharp decrease (regimes II and III, respectively), while the strain hardening rate for the $\mathrm{LA} \perp \mathrm{ED}$ loading decreases monotonically, and its curve undergoes two slope changes: a reduced slope in regime II and a steepened slope in regime III.

\subsection{Mesoscale strain field mapping}

Image sequences are acquired with synchrotron x-ray phase contrast imaging [39-42] during uniaxial compression. The x-ray beam size is about $2.8 \times 2 \mathrm{~mm}^{2}$, slightly larger than the sample cross-section perpendicular to the incident $\mathrm{x}$-rays; the spatial resolution is approximately 4 
(a)
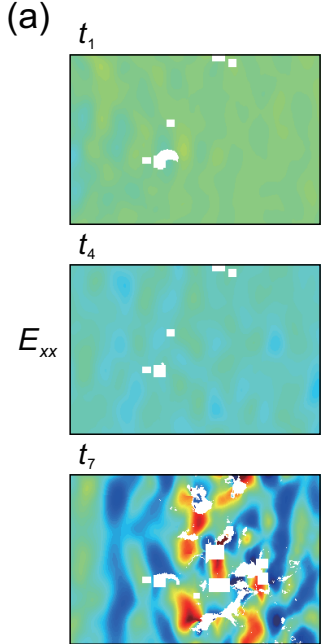

(b)

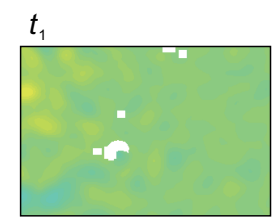

$t_{4}$
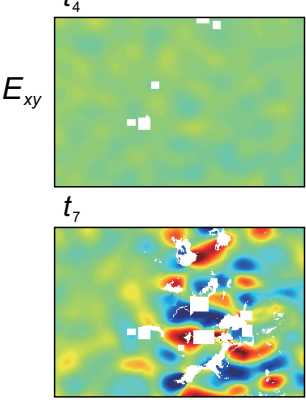

$\overrightarrow{\sqrt{y}}^{x}$
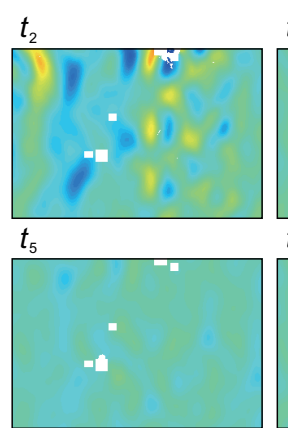

$t_{8}$
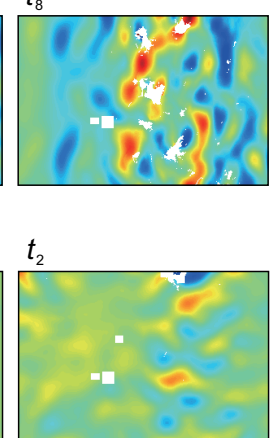

$t_{5}$

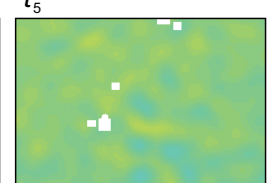

$t_{8}$

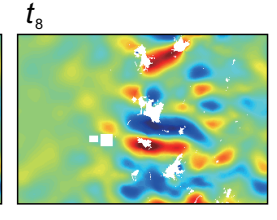

$2 \overline{50 \mu \mathrm{m}}$

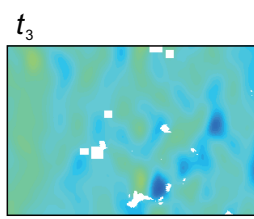

$t_{6}$

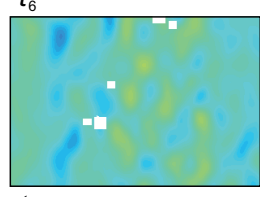

$t_{9}$
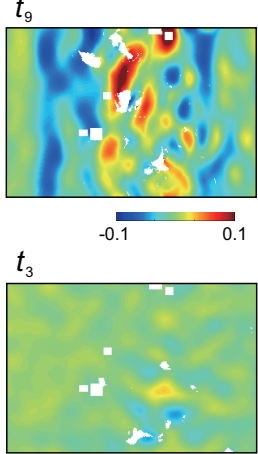

$t_{6}$

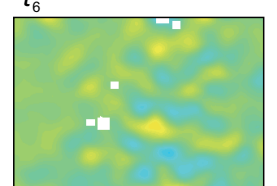

$t_{9}$

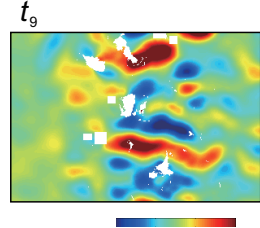

Figure 5: Strain field maps at representative instants $\left(t_{0}-t_{9}\right)$ obtained from XDIC (also refer to Fig. 3) for the LA $\|$ ED loading. (a) Green-Lagrange normal strain $E_{x x}$, and (b) shear strain $E_{x y}$.

$\mu \mathrm{m}$. Fig. 4(a) and (b) show representative x-ray phase contrast images before and during deformation. The x-ray speckles due to surface roughness distribute uniformly, and can be used for image correlation.

To understand the mesoscopic deformation and damage mechanisms, XDIC is performed to map the strain fields. The XDIC method was detailed elsewhere [34]. XDIC "traces" x-ray speckles in x-ray images to resolve displacements by applying image correlation between two adjacen$\mathrm{t}$ frames. The Green-Lagrange normal strains $\left(E_{x x}\right)$ and shear strain $\left(E_{x y}\right)$ are calculated from displacement $(u)$ gradients as

$$
E_{i j}=\frac{1}{2}\left(u_{i, j}+u_{j, i}+u_{k, i} u_{k, j}\right)
$$

where $i, j, k=x, y$. The strain fields, $E_{x x}(x, y)$ and $E_{x y}(x, y)$, for the $\mathrm{LA} \| \mathrm{ED}$ and $\mathrm{LA} \perp \mathrm{ED}$ loading obtained at representative instants as noted in Fig. 3, are shown in

(a)

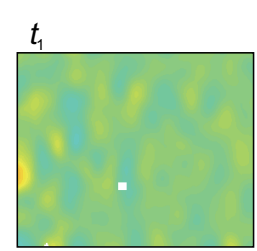

$E_{x x} t_{4}$
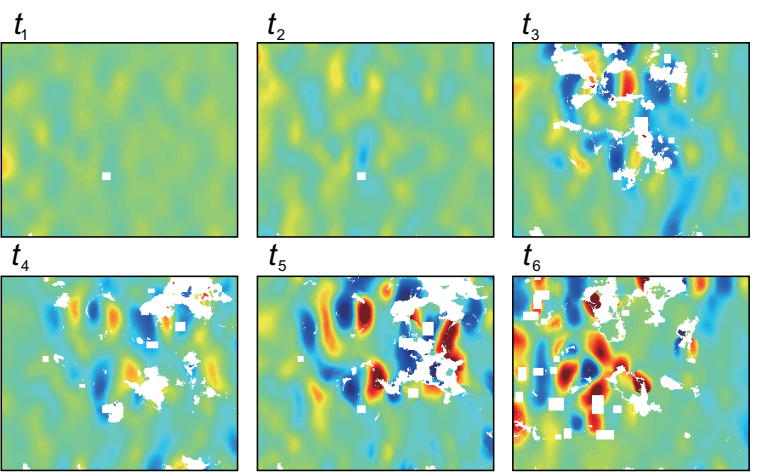

(b)

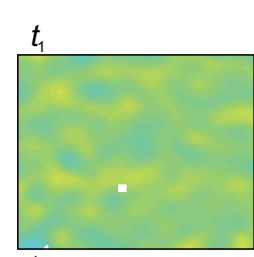

$E_{x y} t_{4}$
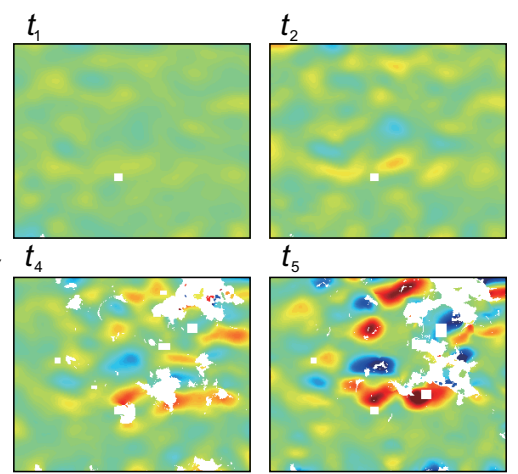

$\sqrt{y}^{x}$
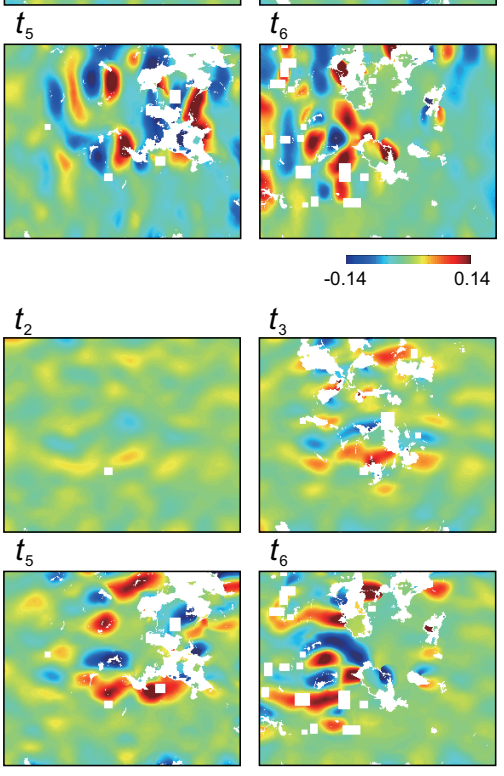

$t_{3}$

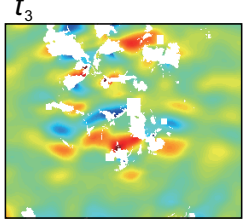

$t_{6}$

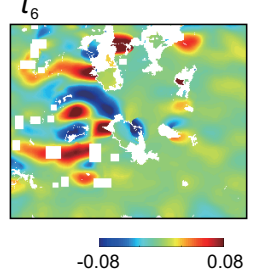

Figure 6: Strain field maps at representative instants $\left(t_{0}-t_{6}\right)$ obtained from XDIC (also refer to Fig. 3) for the LA $\perp E D$ loading. (a) Green-Lagrange normal strain $E_{x x}$, and (b) shear strain $E_{x y}$.

Figs. 5 and 6 , respectively. The areas with poor correlation are replaced with white pixels.

On the basis of mesoscale deformation, four stages can be identified from the normal strain field maps $\left(E_{x x}\right)$ for the LA $\|$ ED loading (Fig. 5(a)): elastic deformation $\left(t_{0}-t_{1}\right.$; Fig. 3), inhomogeneous plastic deformation $\left(t_{1}-t_{2}\right.$; regime I in Fig. 3), homogenization of plastic deformation $\left(t_{2}-t_{6}\right.$; regime II), and accumulation of localized plastic strain $\left(t_{6}-\right.$ $t_{9}$; regime III). The corresponding shear strain fields $\left(E_{x y}\right)$ show similar evolution. The deformation during the initial elastic-plastic transition $\left(t_{1}-t_{2}\right)$ shows slight strain localization. During the homogenization stage $\left(t_{2}-t_{6}\right.$, regime II), compression strain dominates. Strain localization increases rapidly during $t_{6}-t_{9}$ as deformation proceeds into regime III. However, the strain maps $\left(E_{x x}\right.$ and $\left.E_{x y}\right)$ in the LA $\perp E D$ case (Fig. 6) exhibit a different deformation evolution. After the elastic stage $\left(t_{0}-t_{1}\right)$, the LA $\perp$ ED sample experiences continuous local deformation accumulation $\left(t_{1}-t_{6}\right.$; regimes I-III), without the homogenization process observed for the LA $\|$ ED case.

Eventually, both LA $\| \mathrm{ED}$ and LA $\perp$ ED samples undergo out-of-plane shear fracture as identified from in situ $\mathrm{x}$-ray phase contrast images (Fig. 4(b)) and ex situ fractographs, consistent with the large increase of shear strain during $t_{8^{-}}$ $t_{9}(\mathrm{LA} \| \mathrm{ED})$ and $t_{5}-t_{6}(\mathrm{LA} \perp \mathrm{ED})$. 
(a)

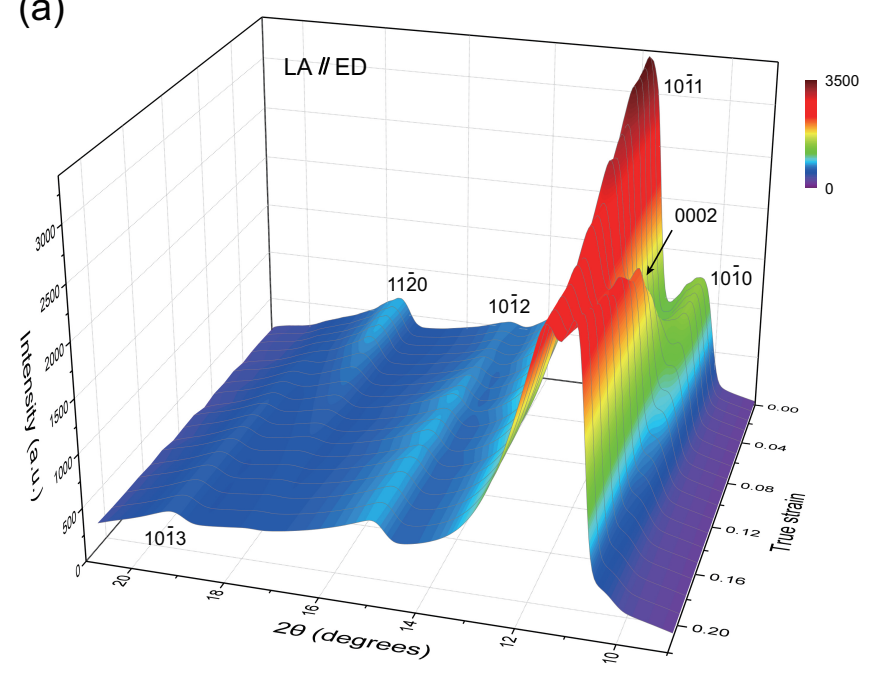

(c)

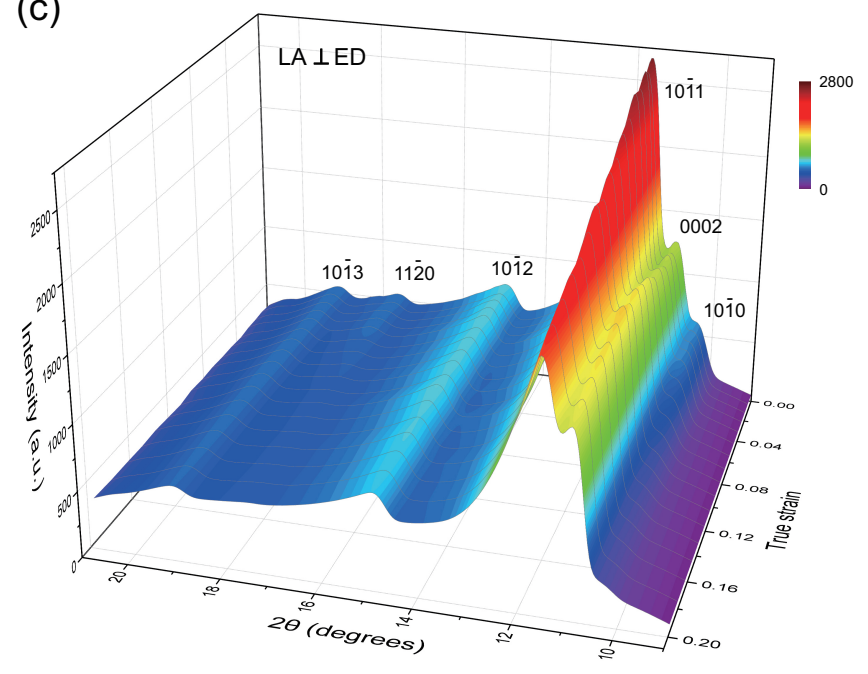

(b)
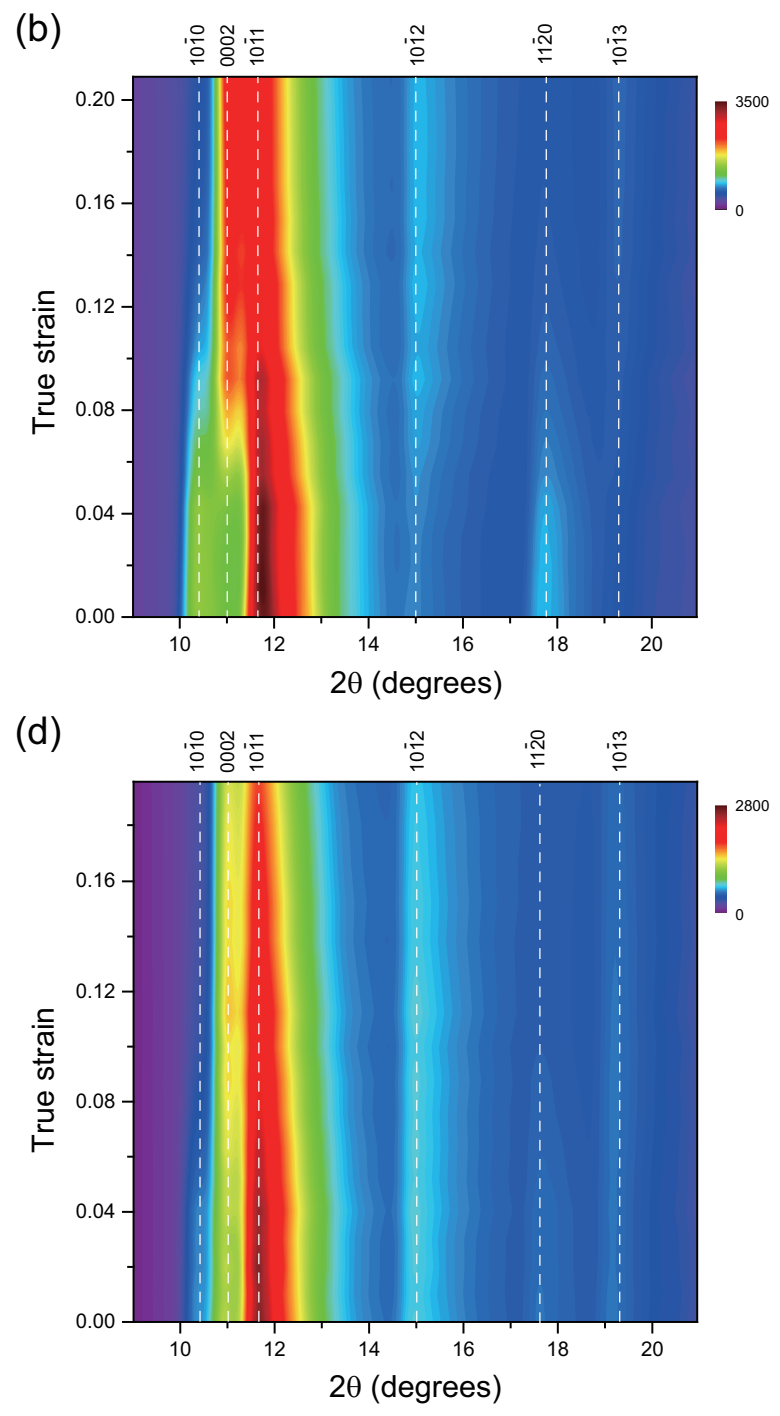

Figure 7: X-ray diffraction patterns as a function of true strain. (a) and (b) LA $\|$ ED. (c) and (d) LA $\perp$ ED. Color bars refer to intensity.

\subsection{Microscale deformation}

In order to investigate microscale deformation including twinning and crystal reorientation, in situ, real time, synchrotron x-ray diffraction measurement is performed, along with ex situ examination of the postmortem samples with EBSD.

\subsubsection{In situ synchrotron $x$-ray diffraction measurement}

Diffraction patterns (intensity versus $2 \theta$ ) as a function of true strain are shown in Fig. 7 for different loading directions: $\mathrm{LA} \| \mathrm{ED}$ and $\mathrm{LA} \perp \mathrm{ED}$. As a result of the initial texture, the undeformed LA $\mid$ ED sample exhibits that the prismatic planes $(\{10 \overline{1} 0\}$ and $\{11 \overline{2} 0\})$ reflections dominates the basal planes $(\{0002\})$ reflection, and the LA $\perp$ ED sample presents contrary results. As deformation proceed$\mathrm{s}$, the $\{0002\}$ reflection for the LA $\| \mathrm{ED}$ sample undergoes rapid growth at $\varepsilon>0.04$, and eventually dominates al1 the other diffraction peaks due to extension twinning, while the $\{0002\}$ peak of the $\mathrm{LA} \perp \mathrm{ED}$ sample experiences a slight increase and then decrease with increasing strain.

\subsubsection{Ex situ EBSD analysis}

As a complement to in situ measurements, postmortem samples are collected and sectioned along the loading direction, and EBSD is utilized to analyze the cross-sections. EBSD inverse pole figure maps, and the corresponding image quality maps and pole figures of the LA $\| \mathrm{ED}$ and $\mathrm{LA} \perp \mathrm{ED}$ samples deformed at various strains, are shown in Figs. 8 and 9, respectively. In the image quality maps (the middle column), three primary types of deformation twins formed during compression are depicted with different colors.

As shown in Fig. 8, with increasing true strain (0.055, 0.080 and 0.117$)$, the $\{10 \overline{1} 2\}$ extension twins thicken quickly, and occupy a significant portion of a grain, or the whole grain, and their number density and area fractions exhibit substantial increase for the LA $\|$ ED sample. When the true strain reaches 0.117 , the area fraction of twins is about $56 \%$, and the $c$-axes of most grains become approximately parallel to LA (Fig. 8(i)). For the LA $\perp$ ED 

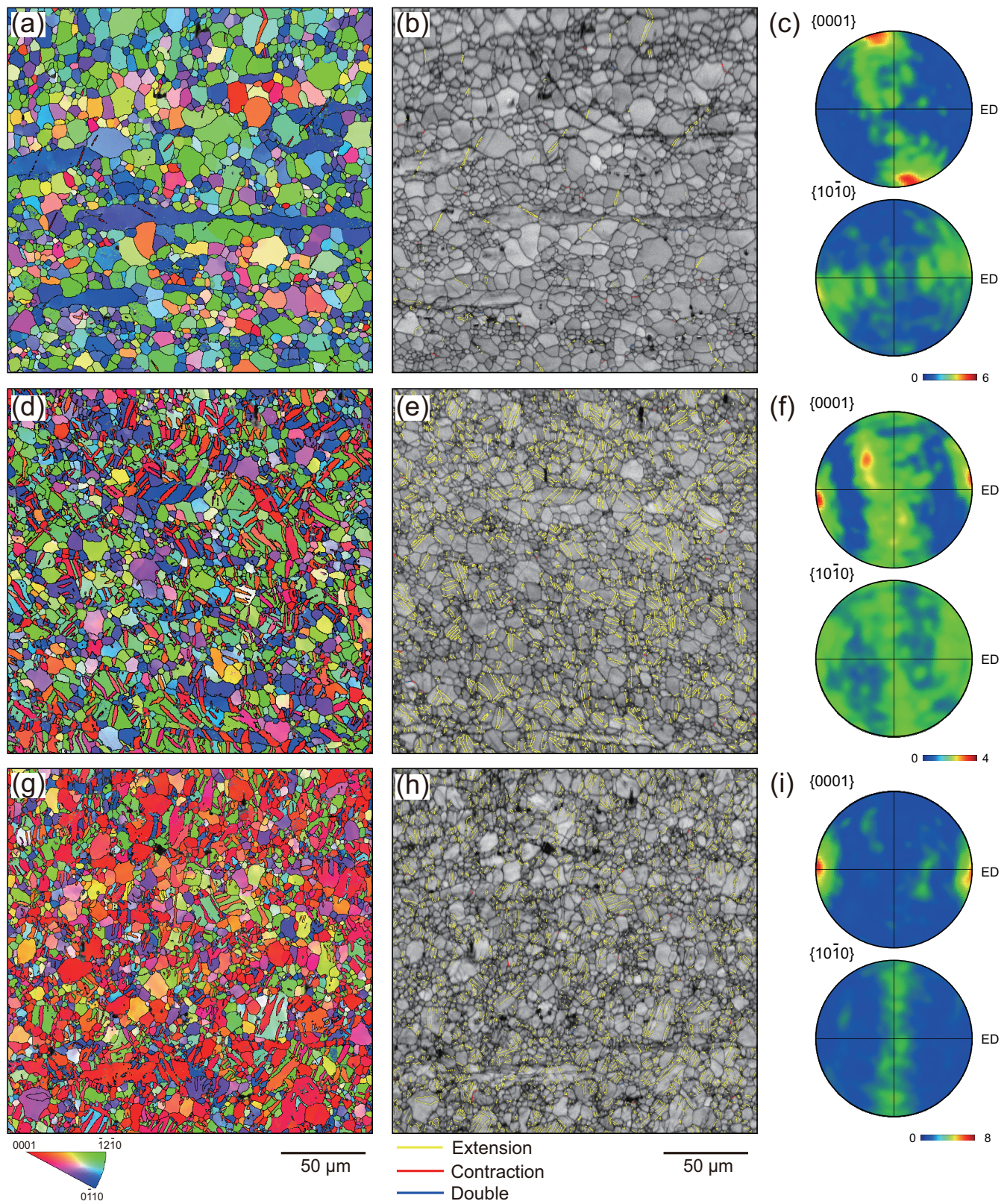

Figure 8: Inverse pole figure maps (left column), the corresponding image quality maps overlaid with twin boundaries (middle column), and pole figures (right column) showing microstructure evolution for the LA $\|$ ED loading. The samples are collected at different true strain levels: (a) 0.055 , (d) 0.080 and (g) 0.117 . In the middle column, yellow curves refer to extension twin boundaries $\left(86.3^{\circ}\langle 1 \overline{2} 10\rangle \pm 5^{\circ}\right)$, red curves to contraction twin boundaries $\left(56.2^{\circ}\langle 1 \overline{2} 10\rangle \pm 5^{\circ}\right)$, and blue curves to double twin boundaries $\left(37.5^{\circ}\langle 1 \overline{2} 10\rangle \pm 5^{\circ}\right)$. The loading axis is horizontal. The $c$-axes of red grains are parallel to ED.

samples, the $c$-axes of major grains are approximately parallel to LA prior to loading. Twinning is negligible at a true strain of $\varepsilon=0.055$. With further compression to 0.080 and 0.117 , abundant $\{10 \overline{1} 2\}$ extension twins are activated in the grains with their $c$-axes initially oriented nearly perpendicular to LA. Eventually, both LA $\|$ ED and LA $\perp E D$ samples show similar textures after uniaxial compression, consistent with previous observations.

\section{Discussions}

In order to understand the effect of $\{10 \overline{1} 2\}$ extension twinning on strain hardening rate during plastic deformation, the intensities of three strong peaks for the LA $\| \mathrm{ED}$ and LA $\perp$ ED samples are presented in Figs. 10(a) and (b), respectively. In regime II, the strain hardening rate increases considerably for the LA $\|$ ED loading (Fig. 3(b)), and the intensity of $\{0002\}$ reflection undergoes a pronounced rise in the meantime. Microstructure deformation shows numerous grains alter their $c$-axis orientations to become parallel to LA by $\{10 \overline{1} 2\}$ extension twinning. In 

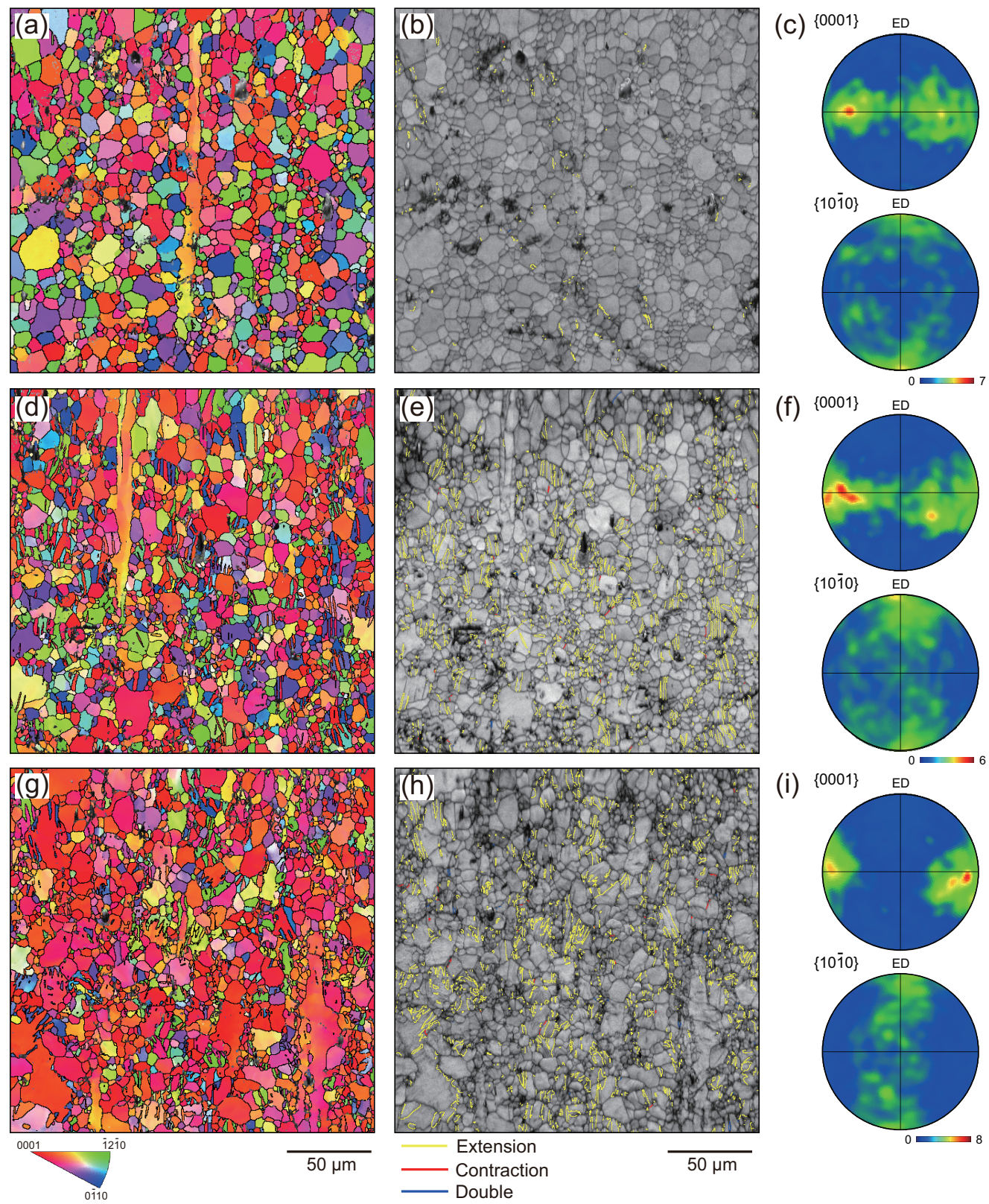

(i)
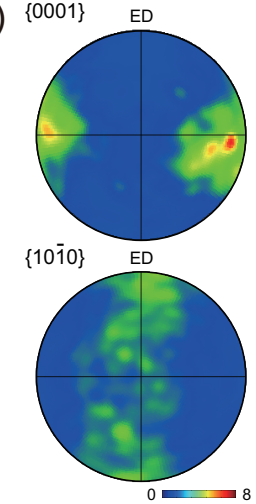

Figure 9: Inverse pole figure maps (left column), the corresponding image quality maps overlaid with twin boundaries (middle column), and pole figures (right column) showing microstructure evolution for the LA $\perp$ ED loading. The samples are collected at different true strain levels: (a) 0.055 , (d) 0.080 and (g) 0.117 . In the middle column, yellow curves refer to extension twin boundaries $\left(86.3^{\circ}\langle 1 \overline{2} 10\rangle \pm 5^{\circ}\right)$, red curves to contraction twin boundaries $\left(56.2^{\circ}\langle 1 \overline{2} 10\rangle \pm 5^{\circ}\right)$, and blue curves to double twin boundaries $\left(37.5^{\circ}\langle 1 \overline{2} 10\rangle \pm 5^{\circ}\right)$. The loading axis is horizontal. The $c$-axes of red grains are perpendicular to the ED.

addition, mesoscale strain field mapping indicates that the LA ||ED sample experiences homogenization in plastic deformation during $t_{2}-t_{4}$ (regime II). $\{10 \overline{1} 2\}$ extension twinning plays an important role in such scenarios. Once twins nucleate within grains, they grow rapidly; twin boundaries are planar and efficient in sweeping across a sample area. Thus, the stress gradients can be released by twinning to achieve homogeneous deformation within a short period of time, and reducing strain localizations effectively boosts the strain hardening rate. In other words, homogenization of plastic deformation as manifested in strain fields is due to abundant $\{10 \overline{1} 2\}$ extension twins, and consequently, gives rise to the pronounced increase in strain hardening rate for the $\mathrm{LA} \| \mathrm{ED}$ loading.

Accompanying slight increase in the $\{0002\}$ peak intensity in regime III for the LA $\|$ ED loading (Fig. 10(a)), moderate activation of $\{10 \overline{1} 2\}$ extension twins also occurs. However, the newborn $\{10 \overline{1} 2\}$ extension twins are not sufficient to dominate plastic deformation which is also achieved by dislocation motion. As a result, the mesoscale strain fields show inhomogeneous plastic deformation and the corresponding macroscopic mechanical behavior ex- 
(a)

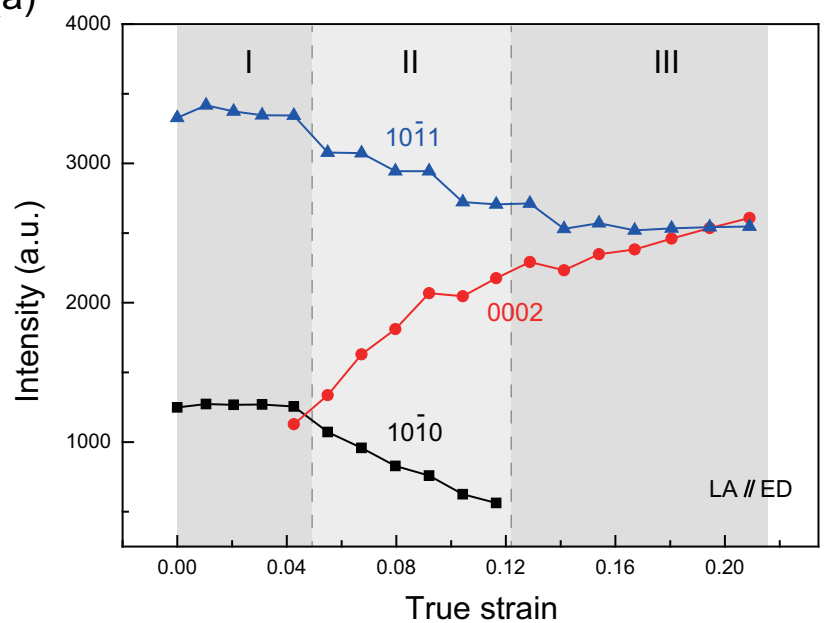

(b)

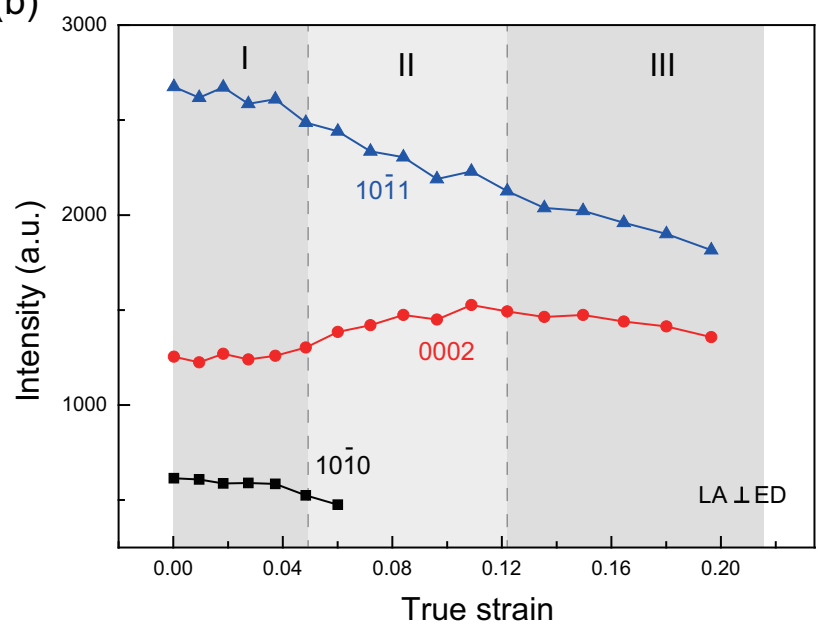

Figure 10: X-ray diffraction peak intensity as a function of true strain for the $\mathrm{LA} \| \mathrm{ED}(\mathrm{a})$ and $\mathrm{LA} \perp \mathrm{ED}$ (b) loading.

hibits a decrease in strain hardening rate.

In addition to twinning, plastic deformation can only be achieved via dislocation motion. With the same plastic deformation in regime II, more twins are observed in the $\mathrm{LA} \| \mathrm{ED}$ sample by comparing to the $\mathrm{LA} \perp \mathrm{ED}$ sample, it can be concluded that more dislocation motion occurs in the $\mathrm{LA} \perp \mathrm{ED}$ sample. Combining macroscopic mechanical behavior of both samples in regime II, it can be found that massive $\{10 \overline{1} 2\}$ extension twins and less dislocation motion lead to the increase in strain hardening rate in the LA\|ED loading, while more dislocation motion and moderate $\{10 \overline{1} 2\}$ extension twins induce slight decrease in strain hardening rate in the $\mathrm{LA} \perp \mathrm{ED}$ loading. In regime II, the $\{10 \overline{1} 2\}$ extension twinning dominates plastic deformation for the LA $\|$ ED loading, while dislocation motion is predominant for the $\mathrm{LA} \perp \mathrm{ED}$ loading. In addition, dislocations nucleated at defects such as grain boundaries induce strain concentrations, which are localized owing to short slide length and pileups. Consequently, mesoscopic strain fields are inhomogeneous (Fig. 6(a)), and the strain hardening rate decreases (Fig. $3(\mathrm{~b})$ ).
In regime III, both $\mathrm{LA} \| \mathrm{ED}$ and $\mathrm{LA} \perp \mathrm{ED}$ samples exhibit inhomogeneous deformation as well as distinct reduction in strain hardening rate, indicating the domination of dislocation motion. The newborn twin boundaries also serve as nucleation sites for elevated dislocation activities, so the strain hardening rate drops faster under the LA $\| \mathrm{ED}$ loading than the LA $\perp$ ED loading. Therefore, inhomogeneous deformation caused by accumulation of dislocations leads to the pronounced decrease in strain hardening rate, countering the effects of deformation twinning.

\section{Conclusions}

An extruded magnesium AZ31 alloy is compressed uniaxially along the $\mathrm{LA} \| \mathrm{ED}$ or $\mathrm{LA} \perp \mathrm{ED}$ direction in conjunction with in situ synchrotron x-ray imaging and diffraction. Multiscale measurements on macroscale stress-strain curves, mesoscale strain fields, and microscale deformation are achieved simultaneously. EBSD is performed on postmortem samples collected at various strains.

- True stress-strain curves and corresponding strain hardening rates for $\mathrm{LA} \| \mathrm{ED}$ and $\mathrm{LA} \perp \mathrm{ED}$ loading show strong anisotropy in deformation.

- For the LA $\|$ ED samples, the formation of abundan$\mathrm{t}\{10 \overline{1} 2\}$ extension twins leads to distinct changes in $\mathrm{x}$-ray diffraction peak intensities compared to the $\mathrm{LA} \perp \mathrm{ED}$ samples with minor twinning.

- When the $\{10 \overline{1} 2\}$ extension twinning dominates plastic deformation, strain field is homogenized, and reducing strain localizations effectively boosts strain hardening rate.

- When dislocation motion dominates plastic deformation, dislocations nucleated at defects induce strain concentrations owing to short slide lengths and pileups. Mesoscopic inhomogeneous deformation induces reduced strain hardening rate.

\section{Acknowledgments}

The authors are grateful to D. K. Qi and Y. Yao for assisting with sample preparation, and the PIMS x-ray team for supporting synchrotron experiments. This work was sponsored in part by the 973 project (No. 2014CB845904), and NSFC (No. 11472227) of China. Use of the Advanced Photon Source, an Office of Science User Facility operated for the U.S. Department of Energy (DOE) Office of Science by Argonne National Laboratory, was supported by the U.S. DOE under Contract No. DE-AC02-06CH11357.

\section{References}

[1] B. L. Mordike, T. Ebert, Magnesium: propertiesapplications_potential, Mater. Sci. Eng. A 302 (2001) 37-45. 
[2] M. Easton, A. Beer, M. Barnett, C. Davies, G. Dunlop, Y. Durandet, S. Blacket, T. Hilditch, P. Beggs, Magnesium alloy applications in automotive structures, JOM 60 (2008) 57-62.

[3] A. Staroselsky, L. Anand, A constitutive model for hcp materials deforming by slip and twinning: application to magnesium alloy AZ31B, Int. J. Plasticity 19 (10) (2003) 1843-1864.

[4] A. Chapuis, J. H. Driver, Temperature dependency of slip and twinning in plane strain compressed magnesium single crystals, Acta Mater. 59 (5) (2011) 1986-1994.

[5] N. V. Dudamell, I. Ulacia, F. Galvez, S. Yi, J. Bohlen, D. Letzig, I. Hurtado, M. T. Pérez-Prado, Influence of texture on the recrystallization mechanisms in an AZ31 $\mathrm{Mg}$ sheet alloy at dynamic rates, Mater. Sci. Eng. A 532 (2012) 528-535.

[6] M. D. Nave, M. R. Barnett, Microstructures and textures of pure magnesium deformed in plane-strain compression, Scripta Mater. 51 (2004) 881-885

[7] S. H. Park, S. G. Hong, C. S. Lee, Activation mode dependent $\{10 \overline{1} 2\}$ twinning characteristics in a polycrystalline magnesium alloy, Scripta Mater. 62 (2010) 202-205.

[8] N. V. Dudamell, I. Ulacia, F. Gálvez, S. Yi, J. Bohlen, D. Letzig, I. Hurtado, M. T. Pérez-Prado, Twinning and grain subdivision during dynamic deformation of a Mg AZ31 sheet alloy at room temperature, Acta Mater. 59 (2011) 6949-6962.

[9] M. R. Barnett, Twinning and the ductility of magnesium alloys: Part II. "Contraction" twins, Mater. Sci. Eng. A 464 (2007) 816.

[10] N. Stanford, M. Barnett, Effect of composition on the texture and deformation behaviour of wrought $\mathrm{Mg}$ alloys, Scripta Mater. 58 (2008) 179-182.

[11] M. Wang, L. Lu, C. Li, X. H. Xiao, X. M. Zhou, J. Zhu, S. N. Luo, Deformation and spallation of a magnesium alloy under high strain rate loading, Mater. Sci. Eng. A 661 (2016) 126131.

[12] S. R. Agnew, Ö. Duygulu, Plastic anisotropy and the role of non-basal slip in magnesium alloy AZ31B, Int. J. Plasticity 21 (2005) 1161-1193.

[13] J. Bohlen, M. R. Nürnberg, J. W. Senn, D. Letzig, S. R. Agnew, The texture and anisotropy of magnesium-zinc-rare earth alloy sheets, Acta Mater. 55 (2007) 2101-2112.

[14] A. A. Salem, S. R. Kalidindi, R. D. Doherty, Strain hardening of titanium: role of deformation twinning, Acta Mater. 51 (2003) 4225-4237.

[15] A. A. Salem, S. R. Kalidindi, S. L. Semiatin, Strain hardening due to deformation twinning in $\alpha$-titanium: Constitutive relations and crystal-plasticity modeling, Acta Mater. 53 (2005) 3495-3502.

[16] M. Lentz, M. Klaus, I. J. Beyerlein, M. Zecevic, W. Reimers, M. Knezevic, In situ X-ray diffraction and crystal plasticity modeling of the deformation behavior of extruded $\mathrm{Mg}-\mathrm{Li}-(\mathrm{Al})$ alloys: an uncommon tension-compression asymmetry, Acta Mater. 86 (2015) 254-268.

[17] M. Knezevic, A. Levinson, R. Harris, R. K. Mishra, R. D. Doherty, S. R. Kalidindi, Deformation twinning in AZ31: influence on strain hardening and texture evolution, Acta Mater. 58 (2010) 6230-6242.

[18] H. El Kadiri, A. L. Oppedal, A crystal plasticity theory for latent hardening by glide twinning through dislocation transmutation and twin accommodation effects, J. Mech. Phys. Solids 58 (2010) 613-624.

[19] D. Sarker, D. L. Chen, Detwinning and strain hardening of an extruded magnesium alloy during compression, Scripta Mater. 67 (2012) 165-168

[20] B. S. Wang, R. L. Xin, G. J. Huang, Q. Liu, Effect of crystal orientation on the mechanical properties and strain hardening behavior of magnesium alloy AZ31 during uniaxial compression, Mater. Sci. Eng. A 534 (2012) 588-593.

[21] Q. Ma, H. El Kadiri, A. L. Oppedal, J. C. Baird, B. Li, M. F. Horstemeyer, S. C. Vogel, Twinning effects in a rod-textured AM30 Magnesium alloy, Int. J. Plasticity 29 (2012) 60-76.

[22] P. Klimanek, A. Pötzsch, Microstructure evolution under compressive plastic deformation of magnesium at different temper- atures and strain rates, Mater. Sci. Eng. A 324 (2002) 145-150.

[23] G. Proust, C. N. Tomé, A. Jain, S. R. Agnew, Modeling the effect of twinning and detwinning during strain-path changes of magnesium alloy AZ31, Int. J. Plasticity 25 (2009) 861-880.

[24] M. R. Barnett, Z. Keshavarz, A. G. Beer, D. Atwell, Influence of grain size on the compressive deformation of wrought $\mathrm{Mg}$ 3Al-1Zn, Acta Mater. 52 (2004) 5093-5103.

[25] H. Fan, S. Aubry, A. Arsenlis, J. A. El-Awady, The role of twinning deformation on the hardening response of polycrystalline magnesium from discrete dislocation dynamics simulations, Acta Mater. 92 (2015) 126-139.

[26] L. Jiang, J. J. Jonas, A. A. Luo, A. K. Sachdev, S. Godet, Influence of $\{10 \overline{1} 2\}$ extension twinning on the flow behavior of AZ31 Mg alloy, Mater. Sci. Eng. A 445 (2007) 302-309.

[27] A. L. Oppedal, H. El Kadiri, C. N. Tomé, G. C. Kaschner, S. C. Vogel, J. C. Baird, M. F. Horstemeyer, Effect of dislocation transmutation on modeling hardening mechanisms by twinning in magnesium, Int. J. Plasticity 30 (2012) 41-61.

[28] S. B. Yi, C. H. J. Davies, H. G. Brokmeier, R. E. Bolmaro, K. U. Kainer, J. Homeyer, Deformation and texture evolution in AZ31 magnesium alloy during uniaxial loading, Acta Mater. 54 (2006) 549-562.

[29] S. H. Choi, E. J. Shin, B. S. Seong, Simulation of deformation twins and deformation texture in an AZ31 $\mathrm{Mg}$ alloy under uniaxial compression, Acta Mater. 55 (2007) 4181-4192.

[30] E. O. Hall, The deformation and ageing of mild steel: III discussion of results, Proc. Phys. Soc. B 64 (1951) 747.

[31] N. J. Petch, The cleavage strength of polycrystals, J. Iron Steel Inst. 174 (1953) 25.

[32] A. Molinari, G. R. Canova, S. Ahzi, A self consistent approach of the large deformation polycrystal viscoplasticity, Acta Metall. 35 (1987) 2983-2994

[33] R. A. Lebensohn, C. N. Tomé, A self-consistent anisotropic approach for the simulation of plastic deformation and texture development of polycrystals: application to zirconium alloys, Acta Metall. Mater. 41 (1993) 2611-2624.

[34] L. Lu, D. Fan, B. X. Bie, X. X. Ran, M. L. Qi, N. Parab, J. Z. Sun, H. J. Liao, M. C. Hudspeth, B. Claus, K. Fezzaa, T. Sun, W. Chen, X. L. Gong, S. N. Luo, Note: Dynamic strain field mapping with synchrotron X-ray digital image correlation, Rev. Sci. Instrum. 85 (2014) 076101.

[35] T. Sun, K. Fezzaa, HiSPoD: a program for high-speed polychromatic x-ray diffraction experiments and data analysis on polycrystalline sample, J. Synchrotron Radiat. 23 (4).

[36] D. Fan, L. Lu, B. Li, M. L. Qi, J. C. E, F. Zhao, T. Sun, K. Fezzaa, W. Chen, S. N. Luo, Transient x-ray diffraction with simultaneous imaging under high strain-rate loading, Rev. Sci. Instrum. 85 (2014) 113902.

37] A. Jain, S. R. Agnew, Modeling the temperature dependent effect of twinning on the behavior of magnesium alloy AZ31B sheet, Mater. Sci. Eng. A 462 (2007) 29-36.

[38] B. Clausen, C. N. Tomé, D. W. Brown, S. R. Agnew, Reorientation and stress relaxation due to twinning: modeling and experimental characterization for Mg, Acta Mater. 56 (2008) 2456-2468.

[39] A. Snigirev, I. Snigireva, V. Kohn, S. Kuznetsov, I. Schelokov, On the possibilities of x-ray phase contrast microimaging by coherent high-energy synchrotron radiation, Rev. Sci. Instrum. 66 (1995) 5486.

40] F. Pfeiffer, T. Weitkamp, O. Bunk, C. David, Phase retrieval and differential phase-contrast imaging with low-brilliance Xray sources, Nat. Phys. 2 (2006) 258-261.

[41] S. N. Luo, B. J. Jensen, D. E. Hooks, K. Fezzaa, K. J. Ramos, J. D. Yeager, K. Kwiatkowski, T. Shimada, Gas gun shock experiments with single-pulse $\mathrm{x}$-ray phase contrast imaging and diffraction at the Advanced Photon Source, Rev. Sci. Instrum. 83 (2012) 073903

[42] J. D. Yeager, S. N. Luo, B. J. Jensen, K. Fezzaa, D. S. Montgomery, D. E. Hooks, High-speed synchrotron X-ray phase contrast imaging for analysis of low-Z composite microstructure, Compos. Part A: Appl. Sci. Manuf. 43 (2012) 885. 

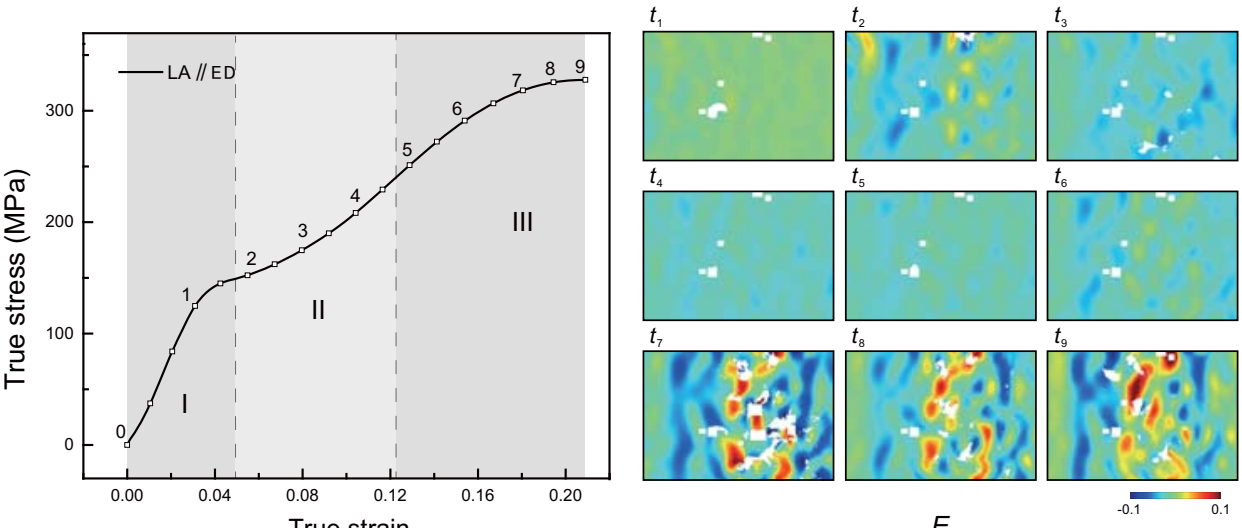

True strain

$$
E_{x x}
$$

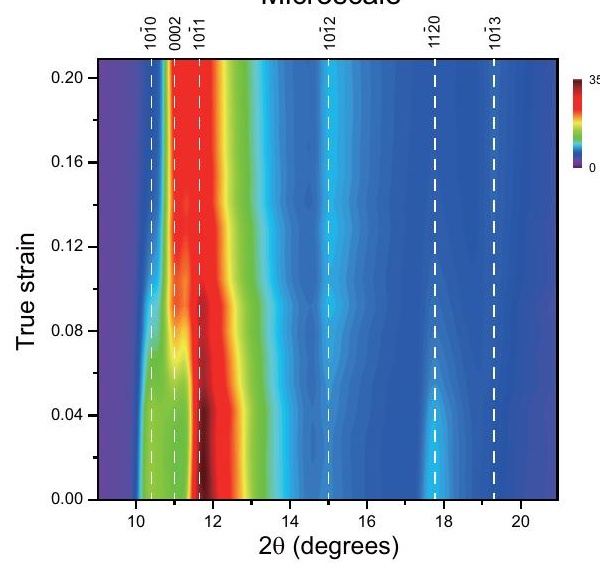

